\title{
ON MEAN VALUES OF DIRICHLET POLYNOMIALS
}

\section{MICHEL WEBER}

Abstract. We show the following general lower bound valid for any positive integer $q$, and arbitrary reals $\varphi_{1}, \ldots, \varphi_{N}$ and non-negative reals $a_{1}, \ldots, a_{N}$,

$$
c_{q}\left(\sum_{n=1}^{N} a_{n}^{2}\right)^{q} \leqslant \frac{1}{2 T} \int_{|t| \leqslant T}\left|\sum_{n=1}^{N} a_{n} e^{i t \varphi_{n}}\right|^{2 q} d t .
$$

Mathematics subject classification (2010): Primary 30B50, Secondary 26D05.

Keywords and phrases: Dirichlet polynomials, mean values, Ingham's inequality, linearly independent sequences, Rademacher sequence, Khintchin-Kahane inequality.

\section{REFERENCES}

[1] K.G. Binmore, A trigonometric inequality, J. London Math. Soc., 41 (1966), 693-696.

[2] A.E. Ingham, A further note on trigonometrical inequalities, Proc. Cambridge Philos. Soc., 46 (1950), 535-537.

[3] A. IvIĆ, The Riemann Zeta-function, Wiley-Interscience Publication, J. Wiley\&Sons, New-York, 1985.

[4] B.S. Kashin, A.A. SAAKyan, Orthogonal Series, Translations of Mathematical Monographs 75, American Math. Soc., 1989.

[5] S.V. Konyagin, H. QuefFÉLec, The translation $\frac{1}{2}$ in the theory of Dirichlet series, Real Anal. Exchange, 27, 1 (2001/2002), 155-176.

[6] H. MONTGOMERY, Ten lectures on the interface between analytic number theory and harmonic analysis, Conference Board of the Math. Sciences, Regional Conference Series in Math. 84, 1993.

[7] I. J. MordeLL, On Ingham's trigonometric inequality, Illinois J. Math., 1 (1957), 214-216.

[8] H. QuefFÉLEC, H. Bohr's vision of ordinary Dirichlet series; old and new results, J. Analysis, 3 (1995), 43-60.

[9] K. Ramachandra, On the Mean-Value and Omega-Theorems for the Riemann Zeta-Function, Tata Institute of Fundamental Research, Bombay, Springer Verlag Berlin, Heidelberg, New-York, Tokyo, 1995, vii+167p. 